\title{
Proceso Estiloides en una Muestra de Radiografías Panorámicas de la Ciudad de Temuco-Chile
}

\author{
Styloid Process in the Panoramics Radiographic Sample of Temuco-Chile City \\ "Ramón Fuentes Fernándes; *Gonzalo Oporto Venegas; "Ivonne Garay Carrasco; \\ ${ }^{* *}$ Luis Bustos Medina; ${ }^{* * *}$ Héctor Silva Mella, \& ${ }^{* * * * *}$ Homero Flores Flores.
}

FUENTES, F. R.; OPORTO, V. G.; GARAY, C. I.; BUSTOS, M. L.; SILVA, M. H. \& FLORES, F. H. Proceso estiloides en una muestra de radiografías panorámicas de la ciudad de Temuco-Chile. Int. J. Morphol., 25(4):729-733, 2007.

RESUMEN: El proceso estiloides es una proyección ósea cilíndrica fina, que mide en promedio $25 \mathrm{~mm}$. Se origina en la porción timpánica del hueso temporal. Este proceso puede encontrarse aumentado en longitud, situación que puede o no manifestarse con dolor. Se estudiaron 968 radiografías panorámicas de sujetos de ambos sexos de edades entre los 12 y 81 años. Fueron medidos los procesos estiloides sobre la radiografía, y se consideraron elongados los que presentaron una longitud mayor a $30 \mathrm{~mm}$. De la muestra, el 3,61\% presentaron procesos estiloides elongados, de éstos, 37,14\% correspondieron a personas de sexo masculino, y $62,86 \%$ de sexo femenino. Al analizar según sexo, los individuos masculinos presentaron procesos más largos que los individuos femeninos, y para ambos sexos se evidenció mayor longitud en el lado derecho (Masculino: longitud derecha 50,2 mm; DS 10,7, longitud izquierda 48,5 mm.; DS 7,9. Femenino: longitud derecha 45,6 mm; DS 7,5; longitud izquierda 42,7; DS 8,3). Se observaron más elongaciones unilaterales que bilaterales, así como elongaciones continuas en comparación a las discontinuas. No existió relación entre edad y elongación de procesos estiloides. Conocer la prevalencia de procesos estiloides elongados es relevante al analizar pacientes con sintomatología dolorosa no solucionada. La presente investigación aporta datos de prevalencia, a los profesionales de la salud, que sería posible encontrar este síndrome.

PALABRAS CLAVE: Proceso estiloides; Síndrome de Eagle, Radiografías panorámicas.

\section{INTRODUCCIÓN}

El proceso estiloides es una proyección ósea cilíndrica fina, que mide en promedio $25 \mathrm{~mm}$. Su origen está en la porción timpánica del hueso temporal. Se relaciona con las arterias carótidas externa e interna, foramen estilomastoideo, nervio facial y la arteria estilomastoidea.

Del proceso estiloídeo se originan tres músculos: estilohioideo, estilogloso y estilofaríngeo, además dos ligamentos: estilohioideo y estilomandibular (Correl et al., 1979).

Esta proyección puede encontrarse aumentada en longitud, situación que puede o no manifestarse con sintomatología. Se ha señalado que entre un $4 \%$ y $28 \%$ de la población posee procesos estiloides elongados, y de ellos, el 4\% evidencia sintomatología (Leile, 1988; Niccollifilho et al., 1986; Sivers \& Joiinson, 1985; Solfanelli et al., 1981; Gonçales et al., 2003; Eagle, 1937; Monsour \& Young, 1986; Castillo et al., 2003; Fernandes et al. 2003).

La elongación del proceso estiloides se presenta más en mujeres (Correl et al.; Watanbe et al., 1998; Gonçales et al., Fernández et al.), pero con mayor longitud en los hombres (Frommer, 1974; Leile). Al análisis de lateralidad, los hallazgos señalan que es más frecuente encontrar elongaciones bilaterales (derecho e izquierdo), con mayor longitud en el lado derecho (Da Silva et al., 2002). El síndrome de Eagle corresponde a una manifestación dolorosa producto de la elongación del proceso estiloides. Esta patología es parte de trastornos temporomandibulares (TTM), los que se enmarcan dentro de los desordenes musculoesqueléticos, que encierran un conjunto de altera-

* Departamento de Odontología Integral, Temuco, Chile.

** Departamento de Salud Pública y Centro de Excelencia CIGES, Temuco, Chile.

*** Departamento de Ciencias Básicas, Unidad de Anatomía, Temuco, Chile.

**** Facultad de Odontología, Universidad de Concepción, Concepción, Chile. 
ciones craneofaciales de etiología multifactorial, referidos a la articulación temporomandibular, músculos masticatorios, cervicales y estructuras asociadas (Ramírez et al., 2005). Muchos de los pacientes con TTM sufren de dolores musculares crónicos en las regiones orofacial, cervical y del oído medio (Ramírez et al.). Un número importante de estos pacientes consultan Otorrinolaringólogos en busca de solución a la sensación dolorosa, pero no siempre hay alteraciones en la regiones del oído, orofaringe o vía aérea superior; de hecho, en ocasiones se presenta en pacientes que han sido previamente tonsilectomizados (Anselmo-Lima et al., 2005).

La cadena estiloidea está formada por el proceso estiloides del hueso temporal, el ligamento estilohioideo y el cuerno mayor del hueso hioides. El aumento en la longitud se describe como la calcificación del ligamento estilohioideo (Ramírez et al.; Anselmo-Lima et al.; Castillo et al.; Louzeiro et al., 2002; Harster, 2001; Melo et al., 2003; Da Silva et al.; Domínguez et al., 2004). Las teorías que explican este fenómeno se basan en origen anómalo congénito (Castillo et al:; Domínguez et al.), óseo endocondral hiperplásico a partir de remanentes de tejidos cartilaginoso y cojuntivo del ligamento (Castillo et al.; Louzeiro et al.; Harster; Domínguez et al. ), metaplasia post traumática o post quirúrgica (Anselmo-Lima et al.; Castillo et al.; Louzeiro et al.; Harster; Melo et al.) y variación anatómica (Castillo et al.). Debido a que la cadena estiloidea se relaciona con estructuras anatómicas, tales como músculos, nervios, arterias, venas y fascia, su aumento de longitud puede llevar a la irritación e inflamación de estas estructuras, con sintomatología dolorosa (Ramírez et al. ; Anselmo-Lima et al.; Castillo et al.; Louzeiro et al.; Harster; Melo et al.; Da Silva et al. ; Domínguez et al.).

En base al síntoma doloroso, se describe el síndrome de Eagle típico, con dolor faríngeo irradiado al oído ipsilateral, salivación intensa, odinofagia y sensación de cuerpo extraño en la orofaringe. Estos síntomas se explican por compresión de terminaciones nerviosas sensitivas de los pares craneanos V, VII, IX y X. El síndrome de Eagle atípico (síndrome de la arteria carótida) se observa con frecuencia en pacientes tonsilectomizados, en los que existe también dolor cervical que se extiende a la cabeza, en el recorrido de las arterias carótidas interna y externa, además de tinitus, vértigo, lipotimias, hiperestesias y mareos. Estaría determinado por presión e irritación de estas arterias y nervios simpáticos de las paredes de estos vasos (Ramírez et al.; Anselmo-Lima et al.; Castillo et al.; Louzeiro et al.; Melo et al.). El diagnóstico está basado en la aparición de la sintomatología señalada, en conjunto al hallazgo imagenológico de un proceso estiloides alargado. No obstante, existen casos en los cuales hay presencia de un proce- so estiloides elongado, sin existir la sintomatología dolorosa, o por otro lado puede ser un hallazgo radiológico con sintomatología inespecífica, no diagnosticada (Castillo et al.; Louzeiro et al.; Harster; Melo et al.; Domínguez et al.). La presente investigación busca establecer la presencia de procesos estiloides elongados, por medio de radiografías panorámicas de pacientes odontológicos, así como su distribución por sexo, patrón de elongación (continuo o discontinuo), lateralidad de la elongación (unilateral o bilateral), la relación entre edad y elongación del proceso estiloides.

\section{MATERIAL Y MÉTODO}

Revisamos 968 radiografías panorámicas de sujetos de ambos sexos, de edad entre 12 y 81 años, en el período comprendido entre los años 2005 y 2006. Éstas fueron tomadas en un Ortopantomógrafo Proline CC Plan Meca. El proceso de revelado se efectuó en una máquina Durr X 24 pro. Se utilizaron placas radiográficas Kodak T- MAT G/ RA.

Los procesos estiloides fueron medidos sobre la radiografía, con un cáliper digital, tomando como referencia la unión con el hueso temporal hasta el ápex. Fueron considerados elongados los procesos estiloideos mayores a $30 \mathrm{~mm}$ (Leile; Moraes et al., 1991; Solfanelli et al.).

Las radiografías que presentaron procesos estiloideos elongados se clasificaron según edad, sexo, lado (unilateral, bilateral) y patrón de la elongación (continuo o discontinuo).

Los resultados fueron tabulados y analizados mediante pruebas estadísticas, utilizando el software STATA ${ }^{\text {TM }}$ 9.0 .
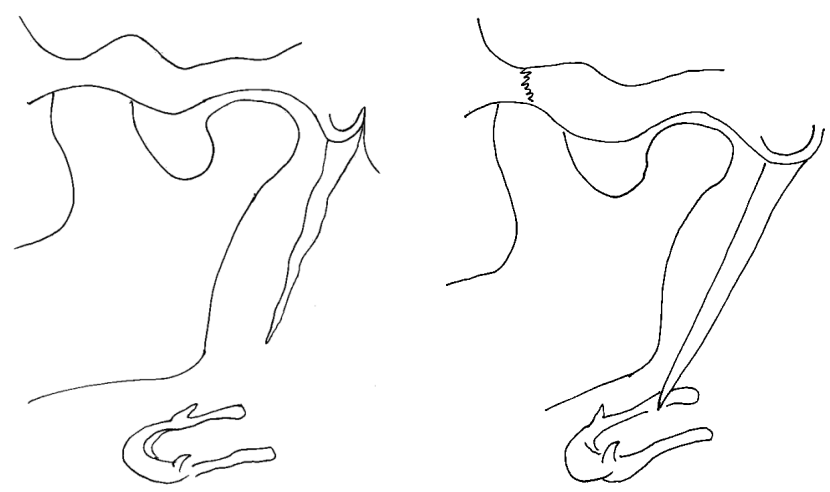

Fig. 1. Patrones de elongación del proceso estiloides. 


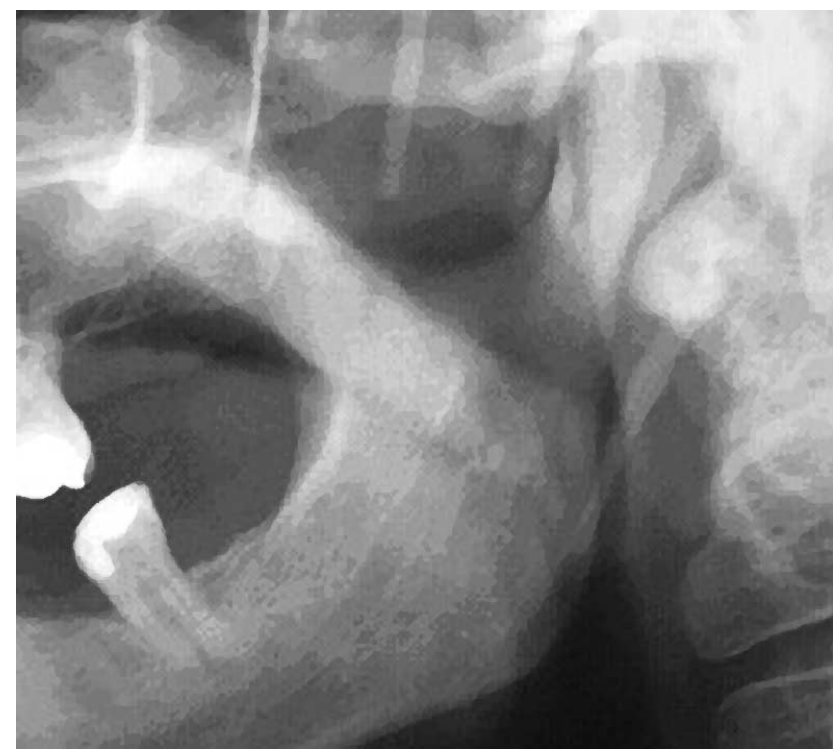

Fig. 2. Radiografía que muestra el proceso estiloides elongado.

\section{RESULTADOS}

Del total de radiografías, $35(3,6 \%)$ en las que se encontró el proceso estiloídeo elongado, $13(37,1 \%)$ correspondían a individuos de sexo masculino y $22(62,8 \%)$ a individuos de sexo femenino.

Los resultados de la distribución de las radiografías con procesos estiloides elongados según sexo, lateralidad, patrón de elongación y edad se encuentran en las Tablas I a V.

\section{DISCUSIÓN}

Los factores que producen la elongación de los procesos estiloides no están claros. La presencia de esta anomalía puede manifestarse con sintomatología o permanecer silente.
Tabla I. Longitud de procesos estiloides elongados en individuos estudiados según lado y sexo.

\begin{tabular}{lccccccc}
\hline Longitud & \multicolumn{3}{c}{ Masculino } & \multicolumn{3}{c}{ Femenino } & $\mathrm{p}$ \\
& $\mathrm{n}$ & $\mathrm{X}$ & $\mathrm{DS}$ & $\mathrm{n}$ & $\mathrm{X}$ & $\mathrm{DS}$ & \\
\hline Lado derecho & 11 & 50,2 & 10,6 & 19 & 45,6 & 7,5 & 0,18 \\
Lado izquierdo & 10 & 48,5 & 7,6 & 22 & 42,7 & 8,3 & 0.07 \\
\hline
\end{tabular}

Prueba t de Student

Tabla II. Patrón de elongación de procesos estiloides en individuos estudiados según sexo.

\begin{tabular}{lccccc}
\hline $\begin{array}{l}\text { Patrón de } \\
\text { elongación }\end{array}$ & \multicolumn{2}{c}{ Masculino } & \multicolumn{2}{c}{ Femenino } & $\mathrm{p}$ \\
& $\mathrm{n}$ & $\%$ & $\mathrm{n}$ & $\%$ & \\
\hline Lado derecho & 9 & 32,1 & 19 & 67,9 & 0,38 \\
Lado izquierdo & 4 & 57,1 & 3 & 42,9 & \\
\hline
\end{tabular}

Prueba estadística de Fisher

Tabla III. Lateralidad de la calcificación de procesos estiloides elongados en individuos estudiados según sexo.

\begin{tabular}{lccccc}
\hline $\begin{array}{l}\text { Lateralidad } \\
\text { de la elongación }\end{array}$ & \multicolumn{2}{c}{ Masculino } & \multicolumn{2}{c}{ Femenino } & $\mathrm{p}$ \\
& $\mathrm{n}$ & $\%$ & $\mathrm{n}$ & $\%$ & \\
\hline Unilateral & 8 & 29,6 & 19 & 70,4 & 0,12 \\
Bilateral & 5 & 62,5 & 3 & 37,5 & \\
\hline
\end{tabular}

Prueba estadística test exacto de Fisher

Tabla IV. Lateralidad de la calcificación de procesos estiloides elongados en individuos estudiados según patrón de elongación.

\begin{tabular}{lccccc}
\hline $\begin{array}{l}\text { Patrón de la } \\
\text { elongación }\end{array}$ & \multicolumn{2}{c}{ Continuo } & \multicolumn{2}{c}{ Discontinuo } & $\mathrm{p}$ \\
& $\mathrm{n}$ & $\%$ & $\mathrm{n}$ & $\%$ & \\
\hline Unilateral & 22 & 81,5 & 5 & 18,5 & \multirow{2}{*}{0,006} \\
Bilateral & 2 & 25,0 & 6 & 75,0 & \\
\hline
\end{tabular}

La prevalencia de elongación del proceso estiloides se encuentra entre el $4 \%$ y $28 \%$ de la población general. (Leile; Nicollifilho et al.; Sivers \& Joiinson; Solfanelli et al.; Gonçales et al.; Eagle; Monsour et al.; Castillo et al.; Fernández et al.) Los resultados de la presente investigación se encuentran bajo el porcentaje encontrado en la literatura, con un $3,6 \%$.

Correl et al.; Watanabe et al.; Gonçales et al. y Fernandes et al., señalaron que esta alteración se presenta con frecuencia en el sexo femenino y es coincidente con lo encontrado en este estudio, donde el $62,8 \%$ de los casos correspondieron a sexo femenino.

La longitud de los procesos estiloides elongados fue mayor en los hombres en ambos lados, en comparación con las mujeres, aunque no estadísticamente significativo (Tabla I). Frommer y Leile, igualmente encontraron mayor longitud en hombres. Cabe señalar que estos estudios sólo se restringieron a los procesos alargados, que coinciden con esta investigación. Da Silva et al., obtienen resultados similares; sin embargo, sus conclusiones fueron obtenidas a partir del promedio general. Es importante destacar que las investigaciones señaladas fueron desarrolladas en material cadavérico y no sobre material imagenológico, como en este estudio. 
Tabla V. Relación entre edad y proceso estiloides elongado en individuos estudiados.

\begin{tabular}{ccc}
\hline Lado & $\mathrm{r}$ & Edad \\
& 0,02 & $\mathrm{p}$ \\
\hline Derecho & $-0,91$ & 0,91 \\
Izquierdo & & 0,29 \\
\hline
\end{tabular}

Tabla VI. Longitud de procesos estiloides elongado según lado en que se presenta alteración en individuos estudiados.

\begin{tabular}{lcccc}
\hline Longitud & $\mathrm{n}$ & Promedio & $\mathrm{DS}$ & $\mathrm{p}$ \\
\hline Derecho & 30 & 47,2 & 8,9 & \\
Izquier do & 32 & 44,5 & 8,5 & 0,227 \\
\hline
\end{tabular}

Prueba estadística t test para dos muestras de igual varianza

El promedio de la longitud de la elongación del proceso estiloides fue mayor en el lado derecho, diferencia no estadísticamente significativa que concuerda con Silva et al. pero menor para ambos lados, en relación al nuestro. En cuanto a la lateralidad, Da Silva et al. señalan mayor presencia de procesos estiloideos elongados bilaterales, en comparación a los encontrados en el presente estudio, que señala que la mayoría de los individuos estudiados, de ambos sexos, presentaron calcificaciones unilaterales. $\mathrm{Al}$ análisis estadístico no hubo diferencias estadísticamente significativas.

El patrón de elongación es un tema escasamente tratado; de hecho, Castillo et al., analizaron TAC, utilizando una nomenclatura que clasifica a estas alteraciones como elongadas (al evidenciar una imagen del proceso calcificado de forma continua) y fragmentadas, con dos variantes de morfología (segmentada y pseudoarticulada) y concluyen que este patrón fragmentado es el más frecuente. Nuestros resultados discrepan con Castillo et al., en relación a ambos sexos; el patrón continuo fue más frecuente, pero no presentó diferencias estadísticamente significativa siendo posible establecer, además, que el patrón continuo de presencia unilateral fue también un hallazgo frecuentemente ob- servado, en comparación al patrón discontinuo de presencia bilateral, siendo estadísticamente significativa la diferencia.

Fernández et al. señalan, en relación a la edad, que la mayoría de las personas con procesos estiloides alargados se encuentran entre los 40 y 60 años de edad. Al analizar la relación edad y elongación de proceso estiloides, la evidencia estadística no estableció una correlación entre estas variables; por lo tanto, para la población estudiada, no existe relación directamente proporcional entre edad y elongación de proceso estiloides.

Los motivos por los que se desarrolla un proceso estiloideo elongado, no han sido del todo dilucidados, sin embargo Castillo et al. señalan que un menor ángulo de inclinación en la elongación podría comprimir el recorrido de los pares craneanos V, VII, IX y X y la vaina carotídea, situación que podría explicar el fenómeno.

La presencia de procesos estiloides elongados en pacientes asintomáticos se relata en la literatura (Leite; Niccollifilho et al.; Sivers \& Jonson; Solfanelli et al.; Gonçales et al.; Eagle; Monsour et al.; Castillo et al.; Fernandes et al.).

Es importante señalar que es necesario continuar las investigaciones al respecto, con el ánimo de lograr establecer claramente las causas. Conocer la prevalencia de procesos estiloides elongados, cobra relevancia al analizar el reporte de casos de pacientes que han consultado a diversos profesionales por sintomatología dolorosa no solucionada (Ramírez et al., Anselmo-Lima et al.). La presente investigación da una aproximación a la frecuencia, que potencialmente es posible encontrar en este síndrome, aportando nuevos datos a los profesionales de la salud e investigadores de las áreas de la Odontología y Otorrinolaringología.

La presente investigación encontró diferencias significativas en la comparación al patrón discontinuo de presencia bilateral.

FUENTES, F. R.; OPORTO, V. G.; GARAY, C. I.; BUSTOS, M. L.; SILVA, M. H. \& FLORES, F. H. Styloid process in the panoramics radiographic sample of Temuco-Chile city. Int. J. Morphol., 25(4):729-733, 2007.

SUMMARY: The Styloid process is a thin cylindrical prolongation of the 2temporal bone. Its mean length is $25 \mathrm{~mm}$. This process could be found longer than average in some people, with or without pain symptomatology. 968 panoramic radiographs have been studied in sexes, male and female, ranging between 12 and 81 years of age. The Styloid processes were measured on radiographs, and were considered elongated with a length higher than $30 \mathrm{~mm}$. Of the studied people, $3.61 \%$ had styloid processes elongated, 37.14\% were male and $62.8 \%$ female. In the analysis by sex, male individuals had longer processes than female, and for both sexes, right styloid processes were longer than left side (Male: right length $50.163 \mathrm{~mm}$., SD 10.65, left length 48.5mm.; SD 7.96. Female: right length 45.6 mm.; SD 7.57, left length 42.74; SD 8.32). Unilateral styloid processes elongated were found more frequently than bilateral, as well as continuous elongations in comparison with discontinuous ones. A relation between age and elongated styloid processes did not exist. To know the prevalence of elongated styloid process becomes important when patients with unsolved pain symptomatology cases are analyzed. This study gives new information to professionals of Dentistry and Otolaryngology areas about the probability to find Eagle's syndrome or elongated styloid process.

KEY WORDS: Styloid process; Eagle's syndrome; Panoramic radiographs. 


\section{REFERENCIAS BIBLIOGRÁFICAS}

Anselmo-Lima, W. T.; Rocha, E. B.; Souza, H. L.; Rodrigues, C. A. \& Aparecido de Oliveira, J. A. Alongamiento do proceso estiloide. A propósito de quatro casos". Rev. Bras. de Otorrinolaringología., 58 (2):124 -9, 2005.

Castillo, C. E.; Mantilla, J. C.; Sandoval, G. P. \& Ramírez, L. M. "Síndrome de Eagle: Tomografía del proceso estiloídeo elongado". Acta De Otorrinolaringología y Cirugía de Cabeza y Cuello., 31(3) 2003.

Correl, R.; Jensen, J.; Taylor, J. \& Rhyme, R: Mineralization of the stylohyoid - stylomandibular ligament complex: A radiographic incidence study. Oral Surg., 48:286, 1979.

Da Silva, H.; Arrunda de Moraes, S. \& Tashiro, T. Análisis Morfométrico de los elementos de la cadena estilohioídea. Rev. Chil. Anat., 20(2):205-10, 2002.

Domínguez A. et al. Alongamiento do processo estilòide (Síndrome de Eagle): Relato de dois casos. Radiol. Bras., 37(5):385-7, 2004.

Eagle, W. W. Elongated styloid process: Report of two cases. Arch Otolaringol., 25:584, 1937.

Fernandes, R. S. M.; Mazzetto, M. O. \& Andrade, C. M. de. Síndrome de Eagle: Protocolo de diagnóstico. JBA., 3(12): 303-9, 2003.

Frommer, J. Anatomic variations in the stylohyoid chain and their possible clinical significance. Oral Surg. Oral Med. Oral Pathol., 38(5):659-67, 1974.

Gonçales, E. S.; nary, H. F.; Alvarez, L. C.; de Oliveira, C. M. \& Stanghuini, V. Síndrome de Eagle: Estudio radiográfico da incidência de processos estilóides alongados. Salusvita Bauru, 22(15):21-4, 2003.

Harster, P. DTM y estiloides. Let me do some speculation about stylohidal process. Ideas y Trabajos Odontoestomatológicos, 2(2)85-9, 2001.

Leile, H. F. Prevalência do processo estiloide alongado en crânios humanos. Rev. Odont. UNESP, 17:145-51, 1988.

Louzeiro, R. S.; Marques, M. F.; Santos, C.A. \& Souza, O. F. Síndrome de Eagle: Avilaçao do tratamento cirúrgico. Rev. Bras. Otorrinolaringol., 69:196-201, 2002.
Melo, R.; Oliveira, M. \& Monteiro. C. Síndrome de Eagle: Protocolo de Diagnóstico. J. Multidisciplinar da Dor Craneofacial., 3(12):303-9, 2003.

Monsour, P. A. \& Young, W. G. Variability of the process and stylohioid ligament in panoramic radiographs. Oral Surg Oral Med Oral Pathol., 61(5): 522-6, 1986.

Moraes, S. et al. Síndrome de Eagle. Relato de um caso. Rev Bras. Odontol., 48(2):30, 1991.

Niccollifilho, W.; Madeira, M. C.; Peake, F. L.; Leite, H.F. \& Simões, S. Prevalence of enlongated styloid process and ossified stylohyoid ligament in adults. A roentgenographic study. Quint. Int. Dent. Dig .Pract Dent., 17(9):581-5, 1986.

Ramírez, L. M.; Sandoval, G. P. \& Ballesteros, L. E. TDM and head symptomatology. Med Oral Patol. CirBucal., 10:E18-E25, 2005.

Sivers, L. E. \& Joiinson, G. K.: Diagnosis of Eagle syndrome. Oral Surg. Oral Med. Oral Pathol. 59:5757,1985

Solfanelli, S. X. et al. Surgical management of symptomatic fractured ossified stylohyoid ligament. Oral Surg., 52(6): 569-73, 1981.

Watanabe, P. A. C.; Campos M. \& Pardini, L. C. Síndrome do processo estilóide alongado (síndrome de Eagle). Rev. Ass. Paulista Cir. Dent., 52(6):487-90, 1998.

Correspondence to:

Dr. Ramón Fuentes Fernándes

Dpto. de Odontología Integral

Universidad de La Frontera

Manuel Montt 112

Temuco - CHILE.

e-mail: rfuentes@ufro.cl.

Recibido : 03-08-2007

Aceptado: 04-10-2007 
